\section{Apert syndrome: Diagnostic and management problems in a resource-limited country}

\author{
Makoura Barro, 1 \\ Yahaya S. Ouedraogo, 1 \\ Fatimata S. Nacro, ${ }^{1}$ Bintou Sanogo, ${ }^{1}$ \\ Solange O. Kombasséré, ${ }^{1}$ \\ Alain S. Ouermi, ${ }^{2}$ Hassane Tamboura, \\ Raymond K. Cessouma, ${ }^{1}$ \\ Boubacar Nacro ${ }^{1}$
}

1Department of Pediatrics, Sourô Sanou University Hospital, Bobo-Dioulasso; 2Department of Pediatrics, Regional Hospital Center of Ouahigouya, Burkina Faso

\begin{abstract}
Apert syndrome or acrocephalosyndactyly is a rare genetic disease characterized by craniofacial dysmorphism and syndactyly of the hands and feet. We report an observation in a 4-month-old female infant, whose father was 65 years old. The infant was admitted to the neonatology of Sourô Sanou University Hospital (Burkina Faso) for respiratory distress in a congenital malformation disorders context with the notion of resuscitation for 10 minutes at birth. Her clinical examination revealed a craniofacial dysmorphism, syndactyly, choanal atresia, a cleft palate and a retardation of the psychomotor development. The paraclinical assessment consisted of a radiograph of the skeleton and a cerebral tomodensitometry confirming bicoronal synostosis and bone syndactyly; an abdominopelvic, cardiac ultrasound didn't reveal any abnormalities; toxoplasmic serology was negative and rubella serology positive. The association of Apert syndrome with positive rubella serology seems fortuitous. Also, the association of choanal atresia and cleft palate has not commonly been reported in Apert syndrome. In the absence of surgical the infant has been followed until 9 months with therapeutic prospects.
\end{abstract}

\section{Introduction}

Apert syndrome, Online Mendelian Inheritance in Man number 101200, is an acrocephalosyndactyly characterized by a craniofacial dysmorphism, syndactyly of the hands and feet and other brain malformations. 1,2 The inheritance of Apert syndrome is autosomal dominant with the locus of a mutation of FGFR2 on chromosome 10q (10q25-26); two most frequent mutations located in exon 7 (Ser252Trp or Pro253Arg) have been found to cause Apert syndrome. ${ }^{2-4}$ It is a rare genetic disease, but sporadic cases are common. ${ }^{2}$ This pathology raises some difficulties of diagnostic confirmation because it makes use of a paraclinical assessment involving carrying out a karyotype and molecular biology. This creates technical and financial problems in our context. Although rare, cases have been reported in Africa and one case of Apert syndrome was previously reported by Yé et al. in Ouagadougou, Burkina Faso. 5 We also report in this study, an observation of an Apert syndrome case of neonatal discovery at Sourô Sanou University Hospital of Bobo-Dioulasso through which we illustrate limits in diagnosis as well as in the management of this pathology in our context.

\section{Case Report}

Y.F is a 4-month-old female infant, the first and only child of her 17-year-old mother, living and apparently healthy; the father is 65 years old; YF has been followed since her birth on $17 / 04 / 2016$ in the Pediatrics Department of Sourô Sanou University Hospital for congenital malformation disorders. As past history, YF, resuscitated at birth for 10 minutes, was hospitalized in neonatology from 17 to $18 / 04 / 16$ for neonatal suffering on congenital malformation disorders type of craniofacial dysmorphism and syndactyly and benefited from antibiotic therapy and a blood test: toxoplasmic serology was negative and rubella serology $(\mathrm{IgG})$ positive. There is a slight developmental delay at 4 months marked by the absence of head holding, the grip hardly appreciable because of syndactyly but she does not try to reach the objects with the hands and the language is limited to a vocalization. There would be no similar cases or other reported malformations in the family. The family tree of YF family is represented by Figure 1 .

The physical examination shows a good general state; a temperature at $37.1^{\circ} \mathrm{C}$, the weight at $6.710 \mathrm{~kg}$ (Weight for Age percentile $=63.5 \%$; Weight For Length percentile $=17.1 \%$ ), the height at $66 \mathrm{~cm}$ (Length For Age percentile $=96.4 \%$ ), the brachial perimeter at $13 \mathrm{~cm}$ and the cranial perimeter at $43 \mathrm{~cm}$ (Head Circumference for Age percentile $=97.1 \%$ ). YF presents a craniofacial dysmorphism composed of a cranial deformity with a flat neck and a domed forehead, a very broad anterior
Correspondence: Makoura Barro, Sourô Sanou University Hospital, Bobo-Dioulasso, Pediatrics Department

01, BP 676 Bobo 01, Burkina Faso.

Tel.: +22670544470

E-mail: barro.mak@fasonet.bf

Key words: Apert; Acrocephalosyndactyly; Burkina Faso.

Acknowledgments: The authors are grateful to the caregivers for their participation in this study.

Contributions: the authors contributed equally.

Conflict of interest: the authors declare no potential conflict of interest.

Funding: none.

Received for publication: 27 June 2019.

Revision received: 19 August 2019.

Accepted for publication: 13 September 2019.

This work is licensed under a Creative Commons Attribution NonCommercial 4.0 License (CC BY-NC 4.0).

(C) Copyright: the Author(s), 2019

Licensee PAGEPress, Italy

Pediatric Reports 2019; 11:8224

doi:10.4081/pr.2019.8224

fontanel extending to the forehead, hypertelorism with telecanthus, a prognathia (Figure 2A). We note bilateral and symmetrical syndactyly of the five fingers giving the appearance of the hand in "muffle" corresponding to the type 3 "pink hand" described by Upton, and feet (Figure 2B,C). There are also abnormalities notably constituted ophthalmological asymmetry of the orbits and prominent right eye (grade I) due to shallow orbits (Figure 2A).

At the Oto-rhino-laryngological level, the examination found noisy breathing with a clinical suspicion of choanal atresia, an isolated alveolar palatal cleft. There is no particular aspect of the otological and cervical examination. The complete physical examination of Y.F including examination of apparatuses revealed no particular aspect apart from the abnormalities mentioned above. X-ray of the skull (Figure 3A) shows coronal synostosis; $\mathrm{X}$-ray of the thorax, of the rest of the skeleton without limbs is normal (Figure 3B). Radiography of the limbs (Figure 3C) found bone syndactyly associated with membranous syndactyly found at clinical examination.

The face and brain scanner show a bicoronal synostosis, a large anterior fontanel with a gaping metopic suture suggestive of cranial bone dysplasia. A grade I 
bilateral exophthalmos is also noted; There is no brain abnormality (Figure 4).

The positive diagnosis of Apert syndrome in YF is based on the following points.

Anamnestic arguments: very advanced paternal age (65 years).

Clinical arguments: characteristic craniofacial dysmorphia, syndactyly of the four limbs and slight developmental delay.

Paraclinical arguments: bicoronal synostosis with exophthalmos at the scanner, bone syndactyly of fingers on X-ray.

The confirmation of the diagnosis by complementary investigations notably the carrying out of the karyotype and the molecular biology looking for an abnormality of the karyotype or a mutation of the coding gene for FGFR 2, located at the level of loci q25 and q26 of the chromosome 10 (Ser252Trp and Pro253Arg) would certainly have comforted us in this diagnosis.

In terms of care and follow-up, YF, aged 4 months, could not benefit from orthopedic surgery or orthognathic surgery because of loss to follow-up at the age of 9 months; surgeons had proposed when the baby was 12 months, an operation was done to correct anomalies of fingers and feet. The baby should check at intervals of 8 weeks for a period of 12 months by the pediatrician (in order to manage feeding issues, developmental, ills...) and intervals of 6 months by ophthalmologist and otolaryngologist. If it was necessary, she could check any time. No consultation in neurosurgery was performed because of a lack of neurosurgeon in our city.

When YF was reviewed at 9 months, she had a slight psychomotor delay. Indeed, YF sat in a tripod, gripping was always difficult to appreciate because of the syndactyly but she tried to reach objects with the hands. The language was limited to sounds in syllables.

\section{Discussion}

According to Skidmore, ${ }^{6}$ there is a possibility of antenatal diagnosis of Apert syndrome. This diagnosis is also possible by amniosynthesis and genetic analysis, but this medical examination is not a common practice in our context. At birth, the morphological abnormalities (skull and limbs) observed in the context of the dysmorphic syndrome are characteristic and suggestive of Apert syndrome as described in YF. These different elements have also been noticed in almost all the observations and have sometimes been the only clinical evidence that some authors have relied upon to

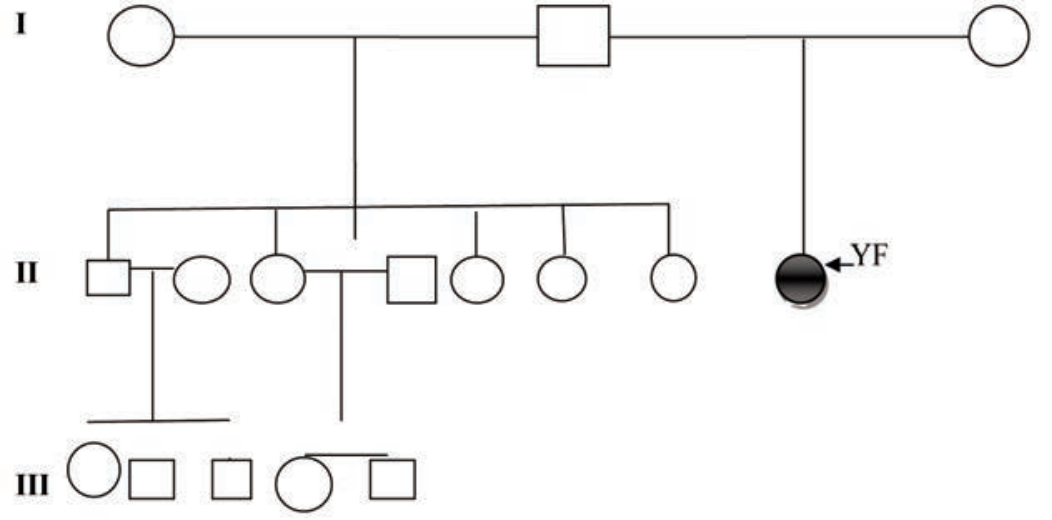

Figure 1. Family tree of YF.
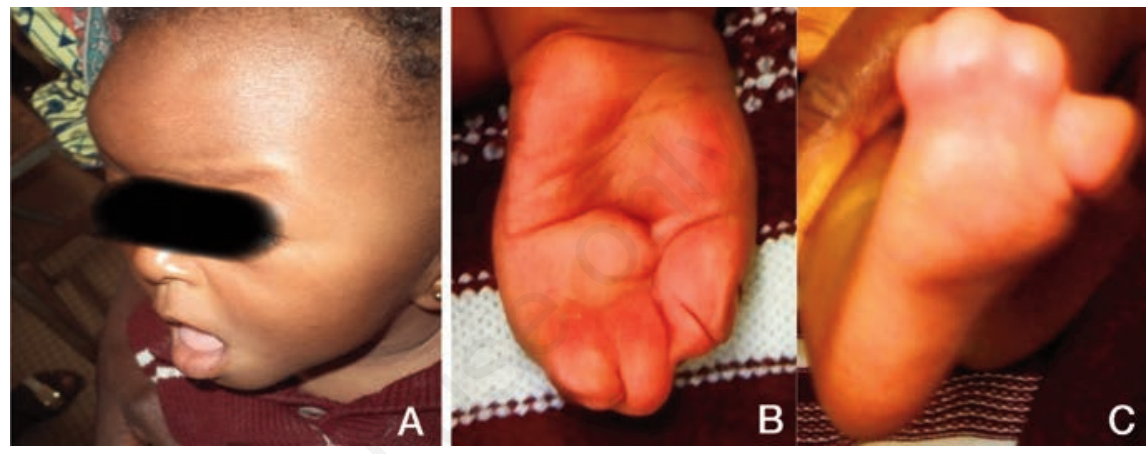

Figure 2. A) Cranio-facial dysmorphia in YF; B) syndactyly of the fingers in YF; C) syndactyly of the toes in YF.
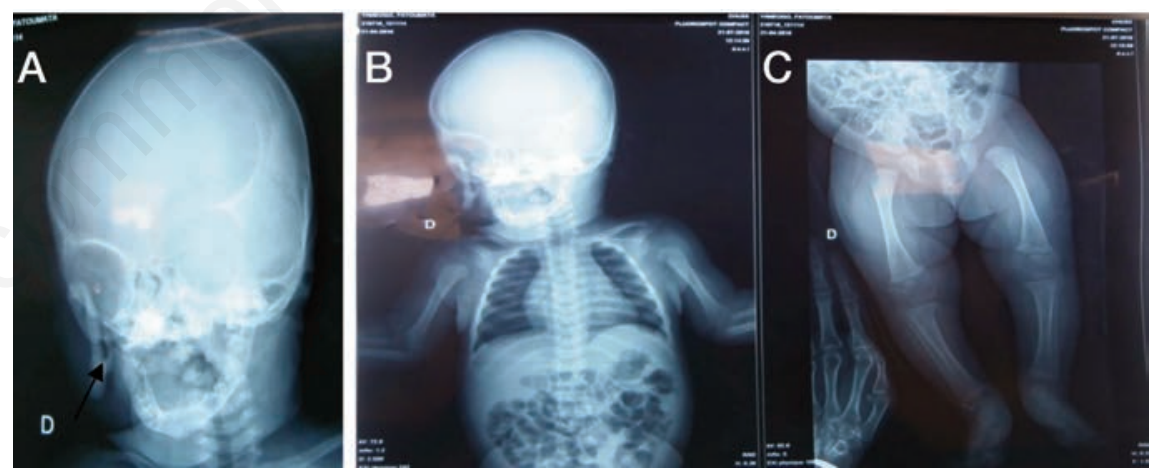

Figure 3. A) X-ray of YF's skull; B) X-ray of YF's thorax and skeleton; C) X-ray of YF's limbs.

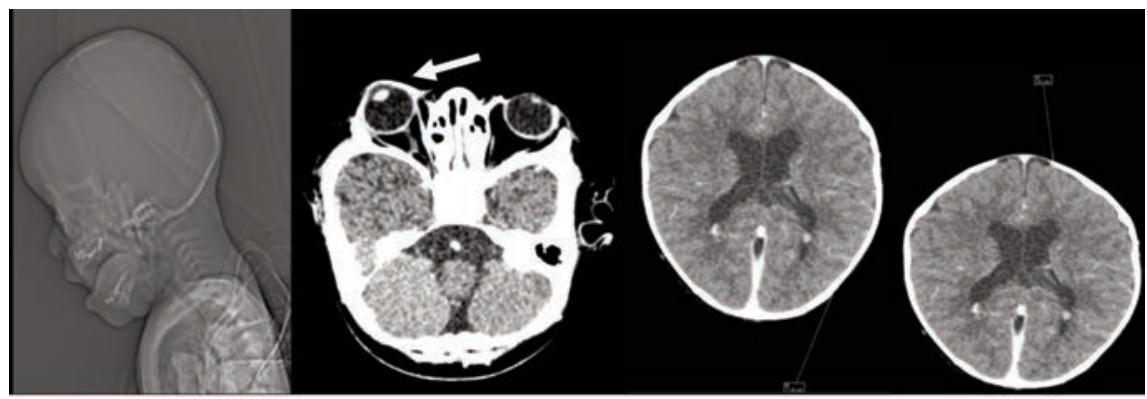

Figure 4. Cerebral CT showing more visible exophthalmia on the right and atresia of the choanal and the absence of parenchymatous lesions. 
make a diagnosis of Apert syndrome.7 However other authors could, in addition to the above-mentioned clinical findings, confirm the diagnosis through molecular biology. 2,4 In fact, Burkina Faso does not have a cytogenetics laboratory for carrying out this checkup, which requires the help of a foreign laboratory, resulting in a cost that is difficult for patients to bear in our context. In addition, some authors have reported the possible role of advanced parental age, particularly that of the father, in the occurrence of Apert syndrome. ${ }^{8}$ The positive serology of rubella without cardiac abnormality and a normal cranial perimeter in YF suggest a juxtaposition of pathologies. Craniofacial anomalies predispose children to airway obstruction. YF had noisy breathing with choanal atresia an isolated alveolar palatal cleft. Association of choanal atresia and cleft palate has not commonly been reported in Apert syndrome. The management of Apert syndrome is complex and requires multidisciplinary collaboration in order to establish a therapeutic schedule considering the abnormalities observed. 4 YF has not received any specific treatment to date because of loss to follow-up at the age of 9 months; so the multidisciplinary follow-up program we had established for $\mathrm{YF}$ in the short term could not be implemented. However, the management program we had established was not optimal for several reasons. Indeed, this child should be treated at a medical center where she will have access to pediatric specialists across the many clinical areas she may need. In our center, there were not pediatric specialists. Additionally, the craniofacial team must maintain close collaboration with genetics, ophthalmology, speech, otolaryngology, audiology and neurosurgery to help provide comprehensive craniofacial care. Most of these specialties didn't exist in our countries.

\section{Conclusions}

This observation made it possible to highlight the limits in diagnosis confirmation namely in the carrying out of molecular biology as well as in the management of this disease in our severity and a significant amount of aesthetic damage. Finally, a special emphasis should be placed on prevention, because in our study, the very advanced paternal age is incriminated in the occurrence of this disease.

\section{References}

1. Lehmeyer JA, Hütsemann W, Mann M, Habenicht R. Transverse soft tissue distraction preceding separation of complex syndactylies. J Hand Surg 2015;17.
2. Brajadenta GS, Sari AIP, Nauphar D, et al. Molecular analysis of exon 7 of the fibroblast growth factor receptor 2 (FGFR2) gene in an Indonesian patient with Apert syndrome: a case report. J Med Case Rep 2019;13:244.

3. Wilkie AO, Slaney SF, Oldridge M, et al. Apert syndrome results from localized mutations of FGFR2 and is allelic with Crouzon syndrome. Nature Genet 1995;9:165-72.

4. Cammarata-Scalisi F, Yilmaz E, Callea $\mathrm{M}$, et al. Clinical and genetic findings of two cases with Apert syndrome. Bol Med Hosp Infantil Mexico. 2019;76:448.

5. Yé D, Sidikou B, Dao L, et al. Syndrome d'Apert : à propos d'un cas au CHUP Charles-de-Gaulle de Ouagadougou. Bull Soc Pathol Exot 2009;102:254-67.

6. Skidmore DL, Pai AP, Toi A, et al. Prenatal diagnosis of Apert syndrome: report of two cases. Prenat Diagn 2003;23:1009-13.

7. Kumar T, Arora N, Puri G, Konidena A. Apert's syndrome: A rare case. J Indian Acad Oral Med Radiol 2016;28:83-5.

8. Glaser RL, Broman KW, Schulman RL, et al. The paternal-age effect in Apert syndrome is due, in part, to the increased frequency of mutations in sperm. Am J Hum Genet 2003;73:93947. 